\title{
TRATAMENTO DA HEPATITE C PELO SISTEMA ÚNICO DE SAÚDE NO BRASIL
}

Raíssa Neves FAGUNDES*

\author{
*Mestre; Farmacêutica; Docente do curso de Enfermagem da Instituição de Ensino Superior Presidente Tancredo \\ Neves- IPTAN, São João Del Rei (MG), brasil.raissanfagundes@ gmail.com.
}

Recebido em: 13/07/2015 - Aprovado em: 13/01/2016 - Disponibilizado em: 30/07/2016

\begin{abstract}
RESUMO
No Brasil, pacientes com infecção crônica pelo vírus da hepatite C são tratados com a terapia dupla ou tripla. Os pacientes em terapia dupla (genótipos 1, 2 ou 3) são tratados com interferonpeguilado alfa $2 \mathrm{a}$ ou alfa $2 \mathrm{~b}$ associado a ribavirina, enquanto os pacientes em terapia tripla (genótipo 1 com fibrose avançada), recebem interferonpeguilado alfa $2 \mathrm{a}$ ou alfa $2 \mathrm{~b}$ associado a ribavirina e telaprevir ou boceprevir. De acordo com o Ministério da Saúde, o objetivo no controle das hepatites virais está em prevenir novas infecções e melhorar a qualidade de vida dos indivíduos portadores desta doença. As últimas décadas foram de grandes mudanças e notáveis conquistas no que se refere à prevenção e ao controle das hepatites virais, porém entre as doenças endêmico-epidêmicas, representa impactante problema de saúde pública. Neste artigo foi realizada uma revisão bibliográfica referente aos medicamentos fornecidos pelo SUS para o tratamento da hepatite $\mathrm{C}$, com o objetivo de analisar a história da dispensação desses medicamentos no sistema público de saúde. No Brasil, há uma necessidade de planejamento no tratamento para hepatite $\mathrm{C}$ para a incorporação de novas drogas, com menor impacto na qualidade de vida, melhor adesão ao tratamento e melhor resposta virológica sustentada. Palavras-chave: Hepatite C. Terapia combinada. Medicamentos excepcionais.
\end{abstract}

\begin{abstract}
In Brazil, currently, patients with chronic hepatitis $\mathrm{C}$ virus are treated with the double or triple therapy. The patients on dual therapy (genotypes 1, 2 or3) are treated with pegylated interferon alpha-2a or alpha $2 \mathrm{~b}$ associated with ribavirin. Patients in triple therapy in patients (genotype1withadvancedfibrosis) receive pegylate dinterferon alfa- $2 \mathrm{a}$ or alpha $2 \mathrm{~b}$ associate dribavirin and telaprevir or boceprevir. According to the Ministry of Health, the main objective in the control of viral hepatitis is to prevent new infections and improve the quality of life of individuals with this disease. The last few decades have brought major changes and outstand in achievements with regard to the prevention and control of viral hepatitis, but among the endemic-epidemic diseases, is impact in public health problem. This article was carried out a literature review relating to medicinal products supplied by SUS for the treatment of hepatitis C, with the aim of analyzing the history of the dispensation of these drugs in the public health system. In Brazil, there is a need for planning the treatment for hepatitis $\mathrm{C}$ for the incorporation of new drugs, less impacton quality of life, shorter treatment time, better adherence to treatment and better sustained virologic response.
\end{abstract}

Keywords: Hepatitis C. Combinationtherapy. Exceptionaldrugs.

\section{INTRODUÇÃO}

O Vírus da hepatite C (VHC) é uma das principais causas da doença hepática crônica no mundo. Estima-se que aproximadamente 115 milhões de pessoas estão infectadas pelo vírus VHC, com uma prevalência global de 1,5\% (GOWER, 2014). A prevalência varia consideravelmente de uma região geográfica para outra, esta discrepância reflete as distintas características epidemiológicas entre as populações (MARTINS, 2011).

$\mathrm{O}$ VHC configura-se como uma das principais causas de cirrose hepática, carcinoma hepatocelular e indicação de transplante hepático em todo o mundo (ARENS, 2001). As principais vias de transmissão de VHC são através do consumo de drogas injetáveis ou 
nasal e, em menor grau, por procedimentos médicos ou cirúrgicos inseguros. O risco de perinatal e da transmissão heterossexuais é baixo, enquanto dados recentes relatam que homossexuais masculinos em atividades promiscuas esta relacionada com a infecção pelo VHC (VAN DE LAAR, 2010).

O tratamento para hepatite $\mathrm{C}$ crônica no Brasil é a terapia dupla e tripla. A terapia dupla se baseia na combinação antiviral com $\alpha$-peginterferon e ribavirina (MARCELLIN, 2011). Atualmente as diretrizes de tratamento da hepatite crônica $\mathrm{C}$ tiveram uma mudança no regime de tratamento para pacientes genótipo tipo 1: a introdução de uma terceira droga no tratamento, os inibidores de serinaprotease, boceprevir e telaprevir (GHANY, 2011). Esses novos medicamentos tem demonstrado um grande potencial na inibição da replicação do vírus da hepatite $\mathrm{C}$ nos genótipos 1 e um significante aumento nas taxas de Reposta Virológica Sustentada (mínimo 20-25\%) em pacientes virgens de tratamento. $\mathrm{O}$ objetivo do tratamento é alcançar a resposta virológica sustentada (RVS) que é definida como uma medida da carga viral indetectável depois de 24 semanas após o fim do tratamento (GHANY, 2009). A obtenção da RVS impede a progressão de fibrose e cirrose e diminui o risco de carcinoma hepatocelular, melhorando assim, a taxa de sobrevivência dos pacientes (ALAVIAN, 2011). Os principais efeitos colaterais desta terapia são: anemia, leucopenia, disfunção da tireóide, desordens neuropsiquiátricas, sintomas gripais, neutropenia (ARENS, 2001).

Os medicamentos constituem parte importante de determinados esquemas terapêuticos previstos no âmbito do SUS e, segundo resultados de estudos recentes, representam significativo gasto adicional dentro do orçamento da maioria da população brasileira (VIEIRA, 2010).

O direito à saúde, previsto na Constituição brasileira, inclui o acesso a medicamentos por meio do Sistema Único de Saúde (SUS). Os programas de assistência farmacêutica do SUS são muitas vezes a única forma de obtenção de medicamentos para grande parte da população brasileira. Dado o caráter de proteção à renda impresso no processo de criação do sistema público de saúde brasileiro, tornou-se necessária à implementação de programas complementares de oferta de fármacos no sistema público de saúde, de forma a possibilitar a continuidade do tratamento, especialmente em casos que requerem o uso de medicamentos de alto custo durante períodos prolongados.

O Programa de Medicamentos de Dispensação Excepcional implementado no âmbito do SUS, apresenta como objetivo a entrega de medicamentos de alto custo a indivíduos que necessitam de tratamento baseado no emprego de tais medicamentos, usualmente fármacos de uso contínuo com elevado valor unitário e direcionados ao apoio 
de pacientes portadores de doenças crônicas (CONASS, 2004).

O Programa de Medicamentos Excepcionais teve início em 1993, porém uma importante ampliação do programa foi iniciada em julho de 2002. O número de medicamentos distribuídos passou de 49 para 87, permitindo que mais 16 problemas de saúde fossem atendidos, como, por exemplo, dor crônica, Osteoporose, Hepatite C, Mal de Parkinson, Doença de Wilson ou Hepatite B em transplantados. Com essa expansão do programa, o número de beneficiários triplicou (BRASIL, 2002).

Segundo o Boletim Epidemiológico, publicado pelo Departamento de DST, Aids e Hepatites Virais em 2012, no Brasil, mais de 82.000 casos de hepatite crônica $\mathrm{C}$ foram confirmados entre os anos de 1999 e 2011. Entre os anos de 2000 e 2010, aproximadamente 14.900 mortes foram atribuídas aos efeitos decorrentes da infecção pelo vírus da hepatite C (VHC) no Brasil. Esse número quase se duplica quando se considera o vírus $\mathrm{C}$ também como condição que contribuiu para a morte, perfazendo um total de 27.231 mortes no mesmo período de dez anos (BRASIL, 2014).

Os medicamentos são essenciais no tratamento da hepatite $\mathrm{C}$ e possuem custo elevado, dentro desse contexto, verifica-se a importância de se analisar a distribuição dos medicamentos para tratamento da hepatite $\mathrm{C}$ no Programa de Medicamentos Excepcionais do SUS, objetivo deste estudo.

\section{TRATAMENTO DA HEPATITE C NO SISTEMA PÚBLICO DE SAÚDE NO BRASIL}

O Programa de Medicamentos Excepcionais teve início em 1982 e fornece medicamentos para o tratamento de doenças específicas. Até 1993, o programa era limitado e fornecia medicamentos apenas a pacientes transplantados e renais crônicos. Apenas em 2002 a Portaria GM/MS n 1.318 incluiu 64 fármacos (incluindo os medicamentos para o tratamento da Hepatite C) em 155 apresentações farmacêuticas, modificando completamente o cenário desse Programa. O Ministério da Saúde divulgou protocolos clínicos e diretrizes terapêuticas com o objetivo de estabelecer os critérios de diagnóstico de cada doença, critérios de inclusão e exclusão de pacientes ao tratamento, as doses corretas dos medicamentos indicados, bem como os mecanismos de controle, acompanhamento e avaliação. $\mathrm{Na}$ ocasião, 30 doenças foram contempladas (CARIAS, 2011).

No âmbito da Portaria GM 1.318/2002, o Ministério da Saúde definiu o valor do cofinanciamento para cada um dos procedimentos padronizados. Por meio do Programa de Medicamentos Excepcionais, os estados tinham a responsabilidade de realizar 
a aquisição dos medicamentos. A dispensação de tais medicamentos devia ser realizado de acordo com as orientações preconizadas nos Protocolos Clínicos e Diretrizes Terapêuticas definidas pelo Ministério da Saúde. Em 2006, o Ministério da Saúde, em pactuação na Comissão Intergestores Tripartite, realizou a revisão do Programa de Medicamentos Excepcionais, por meio da Portaria GM n 2577 de 27 de outubro de 2006. Essa Portaria definiu claramente os objetivos e as responsabilidades dos estados e União em relação ao denominado Componente de Medicamentos de Dispensação Excepcional (BRASIL, 2002).

Em novembro de 2009, a Portaria GM/MS $n^{\circ} 2.981$ constituiu outro marco importante, pois alterou a definição e denominação do programa, que passou a ser tratado como Componente Especializado da Assistência Farmacêutica. Essa transformação beneficiou a população brasileira, que passou a ter acesso gratuito ao tratamento de doenças que oferecem alto risco à saúde (CARIAS, 2011).

Os medicamentos do Componente Especializado da Assistência Farmacêutica estão divididos em três grupos com características, responsabilidades e formas de organização distintas. O Grupo 1 é aquele cujo financiamento está sob a responsabilidade exclusiva da União. É constituído por medicamentos que representam elevado impacto financeiro para o componente, por aqueles indicados para doenças mais complexas, para os casos de refratariedade ou intolerância a primeira e/ou a segunda linha de tratamento e que se incluem em ações de desenvolvimento produtivo no complexo industrial da saúde. Entre eles estão os medicamentos para o tratamento das hepatites B e C (BRASIL, 2002).

A assistência farmacêutica é parte integrante do direito à saúde e prevê o acesso gratuito aos medicamentos por meio de diferentes programas. O Programa de Medicamento de Alto Custo prevê tratamento de custo elevado. Os pacientes que participam desse programa são, por exemplo: pacientes transplantados, portadores de insuficiência renal, hepatites, etc. Os gastos com medicamentos nesse programa têm apresentado crescimento contínuo. Em 2003, foram gastos aproximadamente $\mathrm{R} \$ 1,05$ bilhão e em 2005 estes recursos já somavam $\mathrm{R} \$ 1,92$ bilhão (BRASIL, 2011).

Com o objetivo de ampliar a capacidade de resposta do SUS, foram desenvolvidas ações para enfrentar os problemas relacionados com as hepatites virais em todo território nacional, a finalidade de ampliar a detecção das hepatites virais; reduzir o surgimento de novos casos; além da taxa de mortalidade da hepatite C. Dentro desse contexto, o Sistema Único de Saúde (SUS) tem publicado portarias que especificam procedimentos, medicamentos e 
imunobiológicos utilizados na atenção às hepatites virais, além de instrumentos para seus financiamentos (FERREIRA, 2004).

O Ministério da Saúde em 5 de fevereiro de 2002, criou o Programa Nacional para a Prevenção e o Controle das Hepatites Virais (PNHV), cujos objetivos foram desenvolver ações de promoção, prevenção e assistência aos pacientes com hepatites virais; promover a vigilância epidemiológica e sanitária; ampliar o acesso e incrementar a qualidade e a capacidade instalada dos serviços de saúde em todos os seus níveis de complexidade; bem como organizar, regulamentar, acompanhar e avaliar o conjunto das ações de saúde (FERREIRA, 2004).

Pesquisas revelaram que os ambulatórios de referência para assistência às hepatites têm acesso à exames bioquímicos básicos (transaminases, bilirrubinas e gamaGT), porém exames de biologia molecular são de difícil acesso para a maioria dos estados. Para exames de biologia molecular, a falta de laboratórios com estrutura/equipamentos adequados, a não disponibilidade dos exames pela Secretaria de Estado da Saúde (SES) e a terceirização foram citadas como as principais dificuldades. A biópsia hepática, exame fundamental para a indicação do tratamento na hepatite viral crônica, está disponível em praticamente todos os serviços. Em relação aos medicamentos as principais dificuldades foram: falta de medicamentos, fornecimento pela SES e burocracia no preenchimento de papéis, além da demora nos processos de liberação destes. $\mathrm{O}$ fornecimento do tratamento para hepatite $\mathrm{C}$, com interferonpeguilado + ribavirina foi encontrado 19,2\% dos estados (SBH, 1999).

No Brasil, os medicamentos interferon alfa, interferonpeguilado alfa e ribavirina são fornecidos gratuitamente pelo SUS, através do Componente de Medicamentos Excepcional da Assistência Farmacêutica do Ministério da Saúde. Neste componente são contemplados medicamentos de uso ambulatorial de elevado valor unitário ou que se tornam excessivamente caros em virtude da cronicidade do tratamento. $\mathrm{O}$ acesso a estes medicamentos exige processo individual. Para inclusão, de acordo com o Protocolo de Tratamento, o paciente deve preencher os critérios diagnósticos relacionados na Portaria SVS/ MS n ${ }^{\circ} 34$, de 28 de setembro de 2007.

O Ministério da Saúde disponibiliza os seguintes medicamentos: interferon alfa $2 \mathrm{a}$, interferon alfa $2 \mathrm{~b}$, interferonpeguilado alfa $2 \mathrm{a}$, interferonpeguilado alfa $2 b$ e ribavirina. Dentre os critérios de inclusão para receber interferonpeguilado alfa, o paciente deve ser portador do VHC de genótipo 1. Até setembro de 2007, entre os critérios de exclusão, estava o tratamento prévio sem resposta virológica ou bioquímica adequada, tanto com interferon alfa, como com interferonpeguilado alfa em monoterapia ou associado à ribavirina. A portaria atual contempla o retratamento de 
pacientes, que não obtiveram resposta viral sustentada, submetidos ao tratamento com interferon alfa recombinante. Os pacientes coinfectados com o HIV, virgens de tratamento, independentemente do genótipo e de biópsia devem ser tratados com interferonpeguilado alfa (MINISTERIO DA SAÚDE, 2013). A referida Portaria exclui o tratamento com interferon alfa ou interferonpeguilado alfa em pacientes com as seguintes características:

- tratamento prévio com interferon alfa peguilado (associado ou não à ribavirina);

- consumo abusivo de álcool nos últimos seis meses;

- hepatopatia descompensada;

- cardiopatia grave;

- doença da tireóide descompensada;

- neoplasias;

- diabetemelito tipo 1 de difícil controle ou descompensada;

- convulsões não controladas;

- imunodeficiências primárias;

- homens e mulheres sem adequado controle contraceptivo;

- gravidez (beta-HCG positivo);

- não concordância com o Termo de Responsabilidade.

Além do Componente de Dispensação de Medicamentos Excepcionais, atualmente, um número expressivo de pacientes recorrem ao Poder Judiciário para garantir que o Estado forneça os medicamentos quando eles não preenchem os critérios de inclusão do protocolo. Este fenômeno está desestruturando a política nacional de medicamentos, comprometendo os orçamentos para sua aquisição. Um estudo realizado em Santa Catarina, em 2005, mostrou que interferonpeguilado alfa era um dos itens principais das demandas judiciais por medicamentos. As especificações para a definição do medicamento de escolha, de acordo com o genótipo viral, bem como os critérios de exclusão pelo Componente de Dispensação de Medicamentos Excepcionais, foram as principais causas para as demandas judiciais de fornecimento de interferonpeguilado alfa (PEREIRA, 2006).

Em meados de 2013 o Ministério da Saúde incluiu na lista dos Medicamentos Excepcionais os inibidores de proteases (IPs) de primeira geração boceprevir e telaprevir. A aprovação desses IPs foi concedida exclusivamente para monoinfectados genótipo 1 do VHC e com fibrose avançada (Metavir F3 e F4), ou cirrose hepática compensada. De acordo com a Portaria ${ }^{\circ} 20$, de 25 de julho de 2012, $\quad$ SCTIE/MS ${ }^{17}$. Esses novos medicamentos aumentaram a RVS, porém intensificaram os efeitos adversos ao tratamento, necessitando de um intenso acompanhamento clinico durante o tratamento.

Atualmente há 1,4 a $1,7 \%$ de infectados pelo VHC no Brasil. Cerca de 15,8 mil pessoas no Brasil estão em tratamento pelo SUS. Sendo o Brasil um dos únicos países em 
desenvolvimento no mundo a oferecer diagnóstico, testagem e triagem universal para hepatites virais em sistemas públicos $\mathrm{e}$ gratuitos (BRASIL, 2015).

Os novos medicamentos para hepatite C, os antivirais de ação direta (DDAs) de segunda geração: daclatasvir, simeprevir e sofosbuvir já foram aprovados pela Organização Mundial de Saúde. Esses DAAs tem demonstrado um alto sucesso no tratamento da hepatite $\mathrm{C}$ crônica, visto que esses medicamentos são livre do uso do INF, reduzem o tempo de tratamento para 12 semanas, possuem poucos efeitos adversos e aumentaram as taxas de RVS para $90 \%$. Isto, juntamente com a melhoria da segurança e tolerabilidade, menor duração do tratamento, e uma administração mais simples em comparação com o tratamento à base de interferon, abre-se a perspectiva de fornecer tratamento em escala, inclusive em contextos de recursos limitados (FORD, 2014).

No Brasil o daclatasvir foi o primeiro dos novos medicamentos aprovado pela Anvisa, (publicado a aprovação em 06/01/2015 no Diário Oficial da União), em seguida o simeprevir (11/03/2015), depois o sofosbuvir (30/03/2015) e por último o Viekira Pak (ombitasvir, veruprevir, ritonavir, dasabuvir) aprovado em 22/04/2015. AComissão Nacional de Incorporação de Tecnologia do SUS (CONITEC) aprovou no dia07 de maio de 2015 a colocação em consulta pública o novo PCDT da Hepatite C que inclui os novos antivirais de última geração (daclastavir, simeprevir e sofodbuvir). Se aprovado o PCDT, o SUS estará autorizado a adquirir os medicamentos e o Brasil será o primeiro país do mundo em desenvolvimento a oferecer modalidades de tratamento livres de interferon para a hepatite C de forma universal, pelo sistema público de saúde, a toda população que necessitar do tratamento para a posterior incorporação no SUS (BRASIL, 2015).

A introdução do tratamento oral representa uma extraordinária economia de recursos para o SUS, onde o mesmo orçamento atual que curam 6.000 infectados vai passar a curar 25.000 mil infectados anualmente (BRASIL, 2015).

\section{CONSIDERAÇÕES FINAIS}

"Saúde é um direito de todos e um dever do Estado." Esse trecho da Constituição Brasileira de 1988 não só definiu a saúde como um direito fundamental do ser humano, mas também abriu o caminho para a revolução mais importante na política de financiamento público da saúde no Brasil consideravelmente.

A hepatite $\mathrm{C}$ é considerada uma das doenças mais negligenciadas, tanto no mundo em desenvolvimento e desenvolvidos. Cerca de 115 milhões de pessoas estão cronicamente infectadas pelo VHC em todo o mundo, e 60$70 \%$ delas irão desenvolver doença hepática 
grave, uma das causas mais importantes de morte. A consequência global da infecção por VHC em longo prazo é um impacto negativo sobre a economia da saúde, muito mais do que os custos com prevenção e terapia.

Do ponto de vista clínico, os objetivos primários do tratamento da hepatite $\mathrm{C}$ visam reduzir a progressão da doença e prevenir as complicações da mesma. Todavia, se não houver adesão do paciente a este tratamento, as chances de cura são drasticamente reduzidas.

O tratamento para hepatite $\mathrm{C}$ vai passar por significantes mudanças nos próximos anos, com novas opções de tratamentos orais, com menor tempo de tratamento, menores efeitos adversos e apresentando uma melhora na RVS. A infecção pelo VHC pode ser eliminada nos próximos 15 a 20 anos com estratégias voltadas para tratar e curar infecções atuais, bem como a prevenção de novas infecções.

Dentro desse contexto nota-se a necessidade de planejamento rápido pelo Ministério da Saúde no tratamento da hepatite C. No Brasil, a necessidade de incorporação de novas drogas já aprovados pelo FDA, com menor impacto na qualidade de vida, é premente.

\section{REFERÊNCIAS}

\section{ALAVIAN, S.M; TABATABAEI, S.V;} BEHANAVA, B; MAHBOOBI, N. Optimal duration of treatment for HCV genotype 1 infection in slow responders: A meta-analysis.

Hepat Mon, 11(8), 612-619, 2011.

ARENS M. Clinical relevant sequent-based genotyping of HBV, HCV, CMV and HIV. J

ClinVirol, 22(1), 11-29, 2001.

BRANDÃO, C.M;GUERRA, A.A

JR;CHERCHIGLIA, M.L;ANDRADE, E.I;ALMEIDA, A.M;DA SILVA, G.D;DE QUEIROZ, O.V;FALEIROS,

D.R;ACURCIO,F.D.E.A. Gastos do

Ministério da Saúde do Brasil com Medicamentos de Alto Custo: Uma Análise Centrada no Paciente. Value in health, 14(5), $71-77,2011$.

BRASIL. Boletim Epidemiológico Hepatites Virais Ano III. No. 1. 2012 Ministério da Saúde. Disponível em: http://www.aids.gov.br/publicacao/2012/bolet im-epidemiologico-de-hepatites-virais-2012 [acesso em 6 de junho de 2014].

BRASIL. Ministério Da Saúde. Departamento DST, Aids e Hepatites Virais. Acesso a informações. Disponível em: http://www.aids.gov.br/noticia/2015/anvisaaprova-o-primeiro-dos-tres-medicamentos-donovo-protocolo-de-tratamento-de-hepatatite [acesso em 21/01/2015].

BRASIL. Ministério da Saúde. Portaria 1.318, de 23 de julho de 2002. Dispõe sobre a inclusão de medicamentos na tabela de 
medicamentos excepcionais. Diário Oficial

da União 2002; 23 jul.

CARIAS, C.M; VIEIRA, F.S; GIORDANO,

C.V; ZUCCHI, P. Medicamentos de

dispensação excepcional: histórico e gastos do Ministério da Saúde do Brasil. Rev. Saúde

Pública, 45(2), 1-7, 2011.

CONSELHO NACIONAL DE

SECRETÁRIOS DA SAÚDE (CONASS).

Para entender a gestão do Programa de

Medicamentos de Dispensação em Caráter

Excepcional. Brasília: CONASS, 2004.

FERREIRA, C.T; SILVEIRA, T.R. Hepatites

virais: aspectos da epidemiologia e da

prevenção. Rev Bras de Epidemiol, 7(4), 473-87, 2004.

FORD, N;SWAN, T;BEYER,

P;HIRNSCHALL, G;EASTERBROOK,

PWIKTOR, S. Simplification of antiviral

hepatitis $\mathrm{C}$ virus therapy to support expanded access in resource-limited settings.J Hepatol, 61(1), 132-138, 2014.

GHANY, M.G;NELSON, D.R;STRADER, D.B;THOMAS, D.L;SEEFF, L.B. An update on treatment of genotype 1 chronic hepatitis $\mathrm{c}$ virus infection: 2011 practice guideline by the American Association for the study of liver diseases. Hepatology, 54(4), 1433$1444,2011$.
GHANY, M.G; STRANDER, D.B;

THOMAS, D.L; SEEFF, L.B. Diagnosis, management, and treatment of hepatitis $C$ : an update. Hepatology, 49(4),1335-1374, 2009.

GOWER, E;ESTES, C;BLACH S;RAZAVISHEARER, K;RAZAVI, H. Global epidemiology and genotype distribution of the hepatitis C virus.J Hepatol, 61(1), 45-67. 2014.

MARCELLIN, P;CHOUSTERMAN, M;FONTANGES, T;OUZAN, D;ROTILY, M;VARASTET, M,;LANG, J.P;MELIN, P;CACOUB, P. Adherence to treatment and quality of life during hepatitis $C$ therapy: a prospective, real-life, observational study.

Liver Int, 31(4), 516-524, 2011.

MARTINS, T; NARCISO-SCHIAVON, J.L; SCHIAVON, L.L. Epidemiology of hepatitis C virus infection.Rev Assoc Med

Bras; 57(1),107-117,2011.

MINISTÉRIO DA SAÚDE. Suplemento 1. Protocolo Clinico e Diretrizes Terapêuticas para Hepatite Viral C e Coinfecções, 2013. PEREIRA, J.R. Análise das demandas judiciais solicitando medicamentos encaminhados à Diretoria de Assistência Farmacêutica da Secretaria de Estado da Saúde de Santa Catarina nos anos de 2003 e 2004 (dissertação). 2006.133p.

Florianópolis (SC): Centro de Ciências da 
Saúde da Universidade Federal de Santa

Catarina; 2006.

SOCIEDADE BRASILEIRA DE

HEPATOLOGIA (SBH). Relatório do Grupo

de Estudo da Sociedade Brasileira de

Hepatologia. Epidemiologia da infecção pelo

vírus da Hepatite C no Brasil. GED, 18, 53-

$58,1999$.

VAN DE LAAR, T.J; MATTHEWS, G.V;

PRINS, M; DANTA, M.Acute hepatitis C in

HIV infected men who have sex with men: an emerging sexually transmitted infection.

AIDS, 24(12), 1799-1812, 2010.

VIEIRA, F.S. Assistência farmacêutica no sistema público de saúde no Brasil. Rev

Panam Salud Publica, 27(2), 149-56, 2010. 